\title{
The relationship between long-term changes in plasma B-type natriuretic peptide levels and electrocardiographic findings
}

\author{
Shinji Fukazawa $\cdot$ Koji Teruya $\cdot$ Takamoto Uemura $\cdot$ Tomoko Omine $\cdot$ \\ Tomoko Matsui - Nobuo Takeda $\cdot$ Hiroteru Okamoto $\cdot$ Kaoru Ichikawa \\ Kosuke Tsuchida $\cdot$ Rieko Takemae $\cdot$ Momoo Matsuda $\cdot$ Tooru Tsunoda
}

Received: 26 March 2007/Accepted: 26 December 2007/Published online: 4 April 2008

(C) The Japanese Society for Hygiene 2008

\begin{abstract}
Objectives Changes in B-type natriuretic peptide (BNP) and electrocardiographic (ECG) findings in an adult Japanese population were measured over a 5-year period, and the statistical relationships between these were evaluated. Methods This was a longitudinal analysis in which data were collected on 353 subjects ( 135 men and 218 women) who had undergone general health checks in 1998 and 2003.

Results Data were examined by correlation coefficient and one-way analysis of covariance using repeated measurements. The correlation coefficient for BNP between 1998 and 2003 was $0.622(P<0.0001)$ for the men and $0.557(P<0.0001)$ for women. The changes in BNP over the same period were $13.71 \pm 26.06(P<0.0001) \mathrm{pg} / \mathrm{ml}$ in the men and $20.17 \pm 32.01(P<0.0001) \mathrm{pg} / \mathrm{ml}$ in the women. In 99 men and 145 women who had undergone ECG tests, with both normal and abnormal findings, visual inspections of changes in the ECG findings with respect to BNP changes were performed over the 5-year period. In men, both age and BNP significantly correlated with
\end{abstract}

S. Fukazawa $(\bowtie) \cdot$ K. Teruya $\cdot$ T. Uemura $\cdot$ T. Matsui

N. Takeda $\cdot$ H. Okamoto $\cdot$ K. Ichikawa $\cdot$ K. Tsuchida $\cdot$

R. Takemae $\cdot$ M. Matsuda $\cdot$ T. Tsunoda

Department of Preventive Medicine and Public Health,

School of Medicine, Kyorin University, 6-20-2 Shinkawa,

Mitakashi, Tokyo 181-8611, Japan

e-mail: fkzw@kyorin-u.ac.jp

K. Teruya

Department of Public Health, Faculty of Health Sciences,

Kyorin University, Tokyo, Japan

T. Omine

Department of Health Education, Faculty of Health Sciences,

Kyorin University, Tokyo, Japan changes in ECG findings; however, in women, no significant correlation between BNP and changes in ECG findings was observed.

Conclusions This regional longitudinal study revealed a gender difference in the relationship between long-term changes in BNP and ECG findings, suggesting that a $10 \mathrm{pg} /$ $\mathrm{ml}$ or more increase in BNP in men over a 5-year period may a indicate worsening of cardiac function and the need for intervention.

Keywords BNP $\cdot$ ECG $\cdot$ Long-term change

\section{Introduction}

From the perspective of cardiac disease prevention, the early identification of high-risk groups and prompt and appropriate treatment are important. Following such early intervention, improved prognosis and cost-benefit ratios can be expected [1,2].

B-type natriuretic peptide (BNP) was isolated from the porcine brain in 1988, and subsequent research has shown that BNP is primarily synthesized and secreted in cardiac ventricles [3]. In particular, the level of BNP is known to elevate in response to increased left ventricular volume and pressure load [4-6]. Plasma BNP measurements may be a useful predictor of cardiac function in clinical practice [7]. Recent studies have revealed relationships between BNP and age, BNP and lipids, and BNP and gender as well as the usefulness of BNP in predicting the risk and severity of ischemic heart disease and arrhythmia [8-12]. A few studies have also shown the sensitivity and specificity of BNP in predicting the risk of heart disease in comparison to electrocardiographic (ECG) and echocardiographic findings, but as these have all been one-time measurements 
of BNP, the significance of the findings is still under debate [13-19]. Long-term changes in BNP with respect to metabolic syndrome factors have also been investigated [20], but a relationship to changes in ECG findings has not yet been reported.

We report here our evaluation of the correlation between long-term changes in BNP and ECG findings. In particular, we discuss the usefulness of the long-term measurement of BNP as a preventive strategy and the impact of gender differences on the interpretation of the results.

\section{Subjects and Methods}

\section{Subjects}

Of 894 individuals (339 men, 555 women) who underwent regional health checks in S City, Okinawa, in 1998, 871 (330 men, 541 women) agreed to take the BNP tests. Similarly, of 921 individuals (340 men, 581 women) who underwent similar examinations in 2003, 874 (323 men, 551 women) agreed to take subsequent BNP tests.

Long-term data for comparison between the study cohorts from 1998 and 2003 in terms of changes in BNP were available for 353 individuals. These included 135 men with a mean age $( \pm$ SD) of $65.8 \pm 8.8$ years in 1998 and 218 women with a mean age $( \pm$ SD) of $64.5 \pm 9.2$ years in 1998 . The data from these subjects were analyzed in this study.

The analysis included participants with current or past histories of cardiovascular diseases and endocrine disorders and those being treated with oral medications.

\section{Methods}

General health checks, including a physical examination and laboratory tests, were performed in S City in accordance with the Health and Medical Service Law for the Elderly. Informed consent for taking plasma BNP samples was obtained orally or in writing. More than $95 \%$ of the participants undergoing yearly health checks gave informed consent for additional blood samples.

Plasma BNP levels were measured by radioimmunoassay using monoclonal antibodies specific for human BNP (SHIONORIA BNP assay kit, Shionogi Co., Tokyo, Japan). After the subject rested in a seated position for $5 \mathrm{~min}$, about $2 \mathrm{ml}$ of blood was collected from the antecubital vein into a polyethylene terephthalate tube (containing $1.5 \mathrm{mg} / \mathrm{ml}$ of EDTA-2Na). Samples were centrifuged immediately and stored in a freezer at $-20^{\circ} \mathrm{C}$ until assayed at the Shionogi-Biomedical laboratory. Intraassay and inter-assay coefficients of variation were 3.0-7.6 and $2.0-3.7 \%$, respectively.
A standard resting 12-lead ECG was performed in each subject; this was conducted following the physical examination health checks, with the subject allowed to rest for 510 min before the ECG. The ECG findings were coded based on the Minnesota code and categorized according to the criteria of the Japan National Survey of Cardiovascular Diseases [21]. Based on these criteria, ECG findings were also divided into class I (normal or slightly abnormal) and class II (abnormal A, B, C, or others) (Table 1).

In this study, BNP98 and BNP03 are the BNP levels in 1998 and 2003, and $\triangle \mathrm{BNP}$ is the change in BNP during this 5-year period (BNP03-BNP98).

The question as to whether ECG findings of class I (normal or slightly abnormal) remained class I from 1998 to 2003 or changed from class I to class II (abnormal A, B, $\mathrm{C}$, or others) was also evaluated. Qualified data were available on 99 men [mean age $( \pm$ SD) in 1998: $64.9 \pm 9.3$ years] and 145 women [mean age $( \pm$ SD) in $199863.5 \pm 9.5$ years].

The relationships between BNP98 and BNP03 were evaluated using Pearson's correlation coefficient and partial correlation coefficients with age as a control variable. The significance of the correlation coefficient was assessed by testing the hypothesis that the correlation is zero.

Table 1 Classification of electrocardiogram (ECG) findings

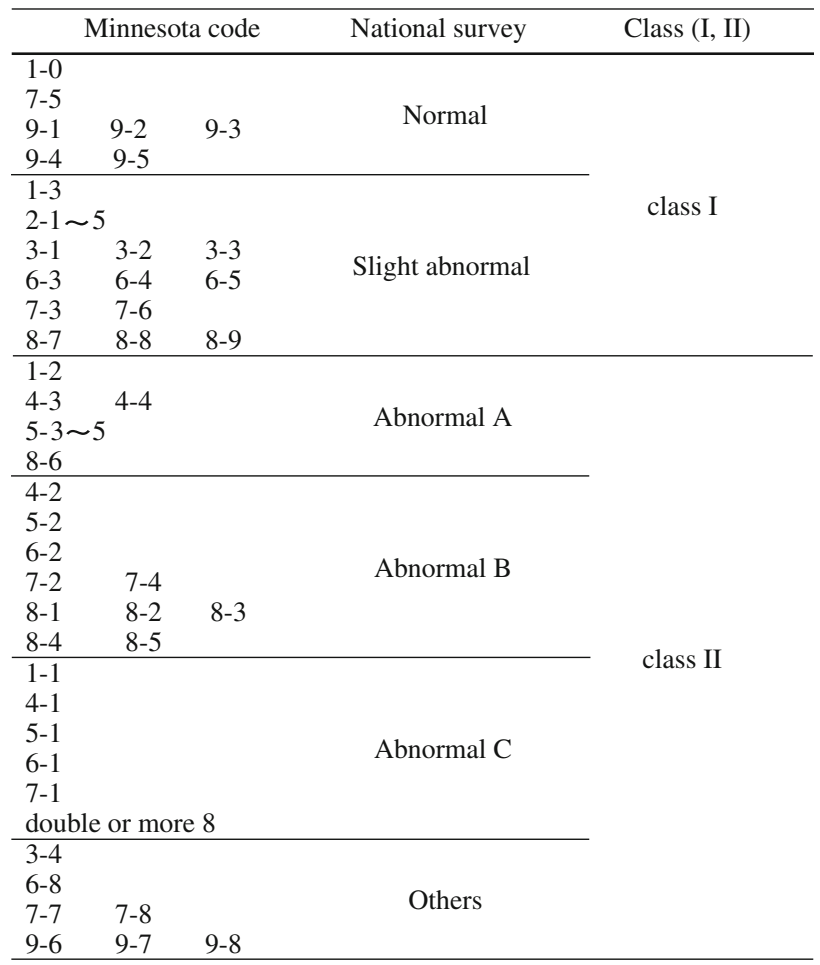

ECG findings were categorized into two groups (class I and class II) according to the criteria of the National Survey of Cardiovascular Diseases [23] 
The change in mean BNP over 5 years was analyzed using a paired $t$ test. Changes in ECG findings and BNP were examined by one-way analysis of covariance (ANOVA) using repeated measurements (paired factors). A probability of $P<0.05$ was considered to be significant. Statistical analysis was all performed using SPSS ver. 12.0 (SPSS, Chicago, IL).

This study was conducted in accordance with the guidelines for epidemiologic research and was approved by the Ethics Committee of Kyorin University School of Medicine.

\section{Results}

The age structure and blood biochemical characteristics of the study subjects are shown in Tables 2 and 3. More than $70 \%$ were of the participants were older than 61 years. To facilitate an evaluation of their biochemical profile, we matched the subjects as closely as possible for body mass index (BMI), systolic blood pressure (BP), and triglycerides (TGs).

We found high diastolic BP and creatinine levels in men, while the BNP, TC, and HDL-C levels were higher in women.

Table 2 Number of subjects classified according to age in 1998

\begin{tabular}{lcc}
\hline Study subjects $(n=353)$ & Men & Women \\
\hline$<50$ years & 10 & 21 \\
$51-60$ years & 20 & 39 \\
$61-70$ years & 69 & 105 \\
$71>$ years & 36 & 53 \\
Total & 135 & 218 \\
\hline
\end{tabular}

Table 3 Blood biochemical characteristics of study population in 1998

\begin{tabular}{lcc}
\hline $\begin{array}{l}\text { Blood biochemical } \\
\text { parameters }\end{array}$ & \multicolumn{2}{c}{ Study subjects $(n=353)$} \\
\cline { 2 - 3 } & Men $(n=135)$ & Women $(n=218)$ \\
\hline BMI & $23.6 \pm 3.1$ & $23.9 \pm 3.3$ \\
Systolic BP & $135.0 \pm 16.6$ & $132.9 \pm 17.1$ \\
Diastolic BP & $77.2 \pm 9.6$ & $74.9 \pm 9.0$ \\
BNP $(\mathrm{pg} / \mathrm{ml})$ & $14.88 \pm 13.46$ & $22.43 \pm 17.20$ \\
Creatinine $(\mathrm{mg} / \mathrm{dl})$ & $1.08 \pm 0.15$ & $0.88 \pm 0.12$ \\
TC $(\mathrm{mg} / \mathrm{dl})$ & $207.3 \pm 32.2$ & $221.9 \pm 34.1$ \\
HDL-C $(\mathrm{mg} / \mathrm{dl})$ & $55.5 \pm 14.4$ & $58.6 \pm 12.9$ \\
TG $(\mathrm{mg} / \mathrm{dl})$ & $128.6 \pm 88.7$ & $129.4 \pm 73.4$ \\
\hline
\end{tabular}

BMI, Body mass index; systolic BP, systolic blood pressure; diastolic $\mathrm{BP}$, diastolic blood pressure; TC, total cholesterol; TG, triglyceride; HDL-C, high-density lipoprotein cholesterol
Figure 1 shows the relationship between BNP in 1998 and 2003. In men $(n=135)$, the correlation coefficient was $0.622(P<0.0001)$, and the partial correlation coefficient, with age as a control variable, was 0.587 $(P<0.0001)$. In women $(n=218)$, these values were $0.557(P<0.0001)$ and $0.508(P<0.0001)$, respectively. The change in mean BNP over 5 years was $13.71 \pm$ $26.06 \mathrm{pg} / \mathrm{ml}$ in men and $20.17 \pm 32.01 \mathrm{pg} / \mathrm{ml}$ in women (Table 4). Paired $t$ test analysis of BNP98 and BNP03 showed a statistically significant increase in BNP $(P<0.0001)$. A gender-related difference in long-term increase was observed.

One-way analysis of covariance using repeated measurements revealed significant correlations in the men between changes in ECG findings and BNP $(P<0.05)$ and between ECG findings and age; in the women, there was a significant correlation between changes in ECG findings and age, but there was no significant correlation between changes in ECG findings and BNP (Table 5; Fig. 2).

In men with ECG findings that remained class I over a 5-year period, the marginal means for BNP (value adjusted for age as a covariate) changed from $13.73 \pm 11.03$ $($ mean $\pm \mathrm{SD})$ to $22.25 \pm 10.29 \mathrm{pg} / \mathrm{ml}$. In men with an ECG that changed from class I to class II, the marginal means for BNP increased from $16.76 \pm 29.71$ to $46.08 \pm$ $27.71 \mathrm{pg} / \mathrm{ml}$. An increase in BNP was observed in women, irrespective of a change in ECG findings (from $22.50 \pm$ 16.14 to $38.20 \pm 16.56 \mathrm{pg} / \mathrm{ml}$ and from $20.81 \pm 26.49$ to $37.86 \pm 27.17 \mathrm{pg} / \mathrm{ml}$, respectively).

In addition to age, analyses were also performed using lipids, BP, and BMI as covariates. These additional factors were found to be of no significance. Only the relationship between BNP and changes in ECG findings in men and the impact of age in men and women were significant.

\section{Discussion}

A relationship between age and BNP has previously been reported in other cross-sectional observation studies. However, few studies have had sufficient sample sizes to permit repeated measurements of BNP as in our study [20], in which BNP was measured twice with a 5-year interval (in 1998 and 2003) in 135 men and 218 women. The correlation coefficient and partial correlation coefficient, with age as a control variable, showed a statistically significant positive correlation; this correlation was slightly stronger in men. In another study of individuals undergoing general health screenings, Kawabe et al. measured BNP two times with a 4-year interval and reported a correlation of 0.588 , but a difference between men and women was not reported [20]. Our results are closely similar to those of 
Fig. 1 Relationship between B-type natriuretic peptide (BNP) in 1998 (BNP98) and 2003 (BNP03). Each plot represents a subject

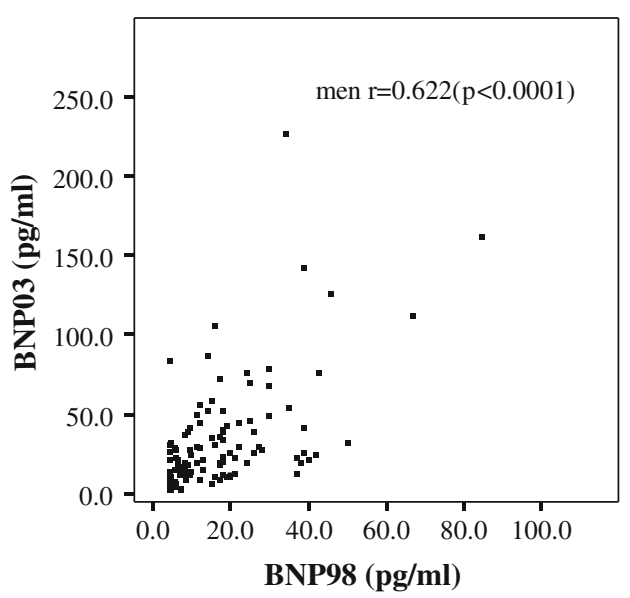

Table 4 Differences in BNP during the 5-year follow-up period (paired $t$ test)

\begin{tabular}{lllll}
\hline & BNP98 $($ Mean \pm SD) & BNP03 (Mean \pm SD) & Significance & $\Delta$ BNP Mean (95\% CI) \\
\hline Men $(n=135)$ & $14.88 \pm 13.46$ & $28.59 \pm 32.79$ & $* * *$ & $13.71(9.18-18.24)$ \\
Women $(n=218)$ & $22.43 \pm 17.20$ & $42.60 \pm 38.24$ & $* * *$ & $20.17(15.90-24.44)$ \\
\hline
\end{tabular}

$* P<0.05, * * P<0.01, * * * P<0.001$

BNP98, B-type natriuretic peptide in 1998; BNP03, BNP in 2003; $\triangle$ BNP, change in BNP in 2003 and 1998; CI confidence interval

$S D$ standard deviation

Table 5 Relationship between BNP and changes in the ECG by analysis of covariance

\begin{tabular}{lrrrrr}
\hline & $\begin{array}{l}\text { Sum of } \\
\text { squares }\end{array}$ & $d f$ & $\begin{array}{l}\text { Mean } \\
\text { square }\end{array}$ & $F$ & Significance \\
\hline Men $(n=99)$ & & & & & \\
$\quad$ Intercept & 519.10 & 1 & 519.10 & 1.85 & \\
Age (covariate) & 3417.68 & 1 & 3417.68 & 12.24 & $* *$ \\
Changes in ECG & 1901.55 & 1 & 1901.55 & 6.81 & $*$ \\
finding & & & & & \\
Error & 26816.69 & 96 & 279.34 & & \\
Women $(n=145)$ & & & & & \\
Intercept & 507.46 & 1 & 507.46 & 1.44 & \\
Age (covariate) & 5632.54 & 1 & 5623.54 & 15.99 & $* * *$ \\
Changes in ECG & 28.02 & 1 & 28.02 & 0.80 & \\
$\quad$ finding & & & & & \\
Error & 49934.90 & 142 & 351.65 & & \\
\hline
\end{tabular}

$* P<0.05, * * P<0.01, * * * P<0.001$

Kawabe et al. and, in addition, we found a slightly higher correlation in men than women.

The paired $t$ test analysis showed a significant long-term change with an increase in BNP. We observed a larger increase in women than in men. In a cross-sectional study among middle-aged women, Chang et al. [21] suggested that the concentration of free testosterone was inversely associated with circulating BNP. This may be related to the higher $\triangle \mathrm{BNP}$ seen in aged women in our study.
Our study demonstrates not only a relationship between age and BNP, as has already been shown in cross-sectional studies, but also confirms, by long-term observation in a regional population, an increase in BNP associated with aging.

Individuals who showed a remarkable renal function disorder value were excluded from this study. Accordingly, we considered that the influence of abnormal creatinine levels on BNP measurements was low.

Among the 871 participants in 1998, there was no obvious difference in health status between the 353 subjects and 518 residuals (data not shown). The health check conditions (place, season, period) and the method of storage or transportation of the specimens to the laboratory were similar enough not to be responsible for the remarkable increase in BNP levels between the two measurements (5 years). Although some studies have shown a slightly lower value of BNP, one reason for this may be the inclusion in these studies of healthy younger subjects. The obtained baseline values for BNP in our study in 1998 $(n=871)$ and $2003(n=874)$ among an elderly, almost healthy population were well within the range of the major study by Redfield et al. (data not shown) [8].

An earlier study reported BNP in participants of a health check program, and serial changes were also observed, but, to date no study has examined 353 almost healthy individuals. We carried out statistical analysis as a covariate of age in this study, but we did not classify the participants into age groups. However, individuals with more than 
Fig. 2 Estimated marginal means of BNP according to changes in the electrocardiogram $(E C G)$

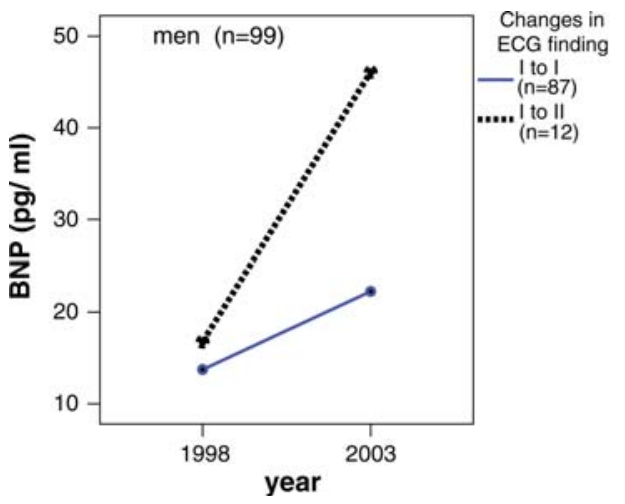

$10 \mathrm{pg} / \mathrm{ml} \Delta \mathrm{BNP}$ were nearly always older than 60 years. Participants taking medication or with left ventricular hypertrophy were not excluded from our study and, therefore, they could possibly represent a factor in the raised BNP concentrations [22].

We examined whether those with class I ECG findings in 1998 remained class I or changed from class I to class II over the 5-year period. A gender-related difference was observed: men with a change in ECG findings from class I to class II showed a significantly greater increase in BNP than those who remained class I. In women, however, there was almost no difference between these subgroups. In both men and women, age had a significant impact on the relationship between the changes in BNP and ECG findings.

We have demonstrated a correlation between long-term changes in BNP and changes in ECG findings and, in addition, shown a gender difference in this relationship. A recent study by Chang et al. suggested that a decreased free testosterone level among women was associated with higher BNP levels. This may help to explain the genderrelated differences and gender-related serial changes in BNP levels among elderly subjects in our study [21].

Our study evaluated changes in ECG findings from class I to class II from the standpoint of prevention. The limited data from patients with class II findings in 1998 were also analyzed, but no clear relationship between BNP and changes in ECG findings could be observed.

Our study was conducted among participants undergoing regional health checks in S City; thus, subjects were generally healthy enough to participate in routine health checks. Also, the subjects included patients with a history of cardiovascular diseases or receiving treatment; therefore, the study was not limited to healthy middle-aged adults. However, participants undergoing such evaluations in outpatient settings tend to have relatively mild diseases, which may further increase the significance of our results.

Our analysis was limited to subjects with available data from both health checks with a 5-year interval; however, among participants with data from only one health check, either 1998 or 2003, the results for age and BNP did not markedly differ from the results in the analyzed subjects. This also validates the appropriateness of our study design.

The aim of our study was cardiovascular disease prevention. We realize that the climate and dietary factors in $\mathrm{S}$ City in Okinawa, where the study was conducted, may differ from other regions in Japan. Our study subjects were relatively elderly, with a mean age in 1998 of 65.8 years in men and 64.5 years in women. The results may help increase awareness of cardiovascular diseases in this group.

Many types of biological data, including BNP, can show a bias in the distribution of values. In our study, we also performed analyses using logarithmic transformation and nonparametric approaches, but there were no marked differences in overall significance. In addition, such statistical considerations are not usually mentioned in the related literature; therefore, for easier interpretation, we have presented the results of the parametric analysis only.

Waveform analyses of ECG findings were coded using the Minnesota code, which is internationally accepted. We also categorized the ECG findings according to the classification of the Japan National Survey of Cardiovascular Diseases. Electrocardiogram findings that can be related to BNP include arrhythmias and myocardial ischemia. These effects may have led to some differences, but a stratified analysis for each finding was not performed. In our study with a relatively healthy population, recruiting sufficient subjects with abnormal findings to permit stratified analysis was difficult. This might be more feasible if the study were performed in a cardiovascular outpatient center.

The risk of cardiovascular disease increases with aging, and BNP is known to be higher in older persons. In this regional study, we also found an increase in BNP with aging. In addition, long-term measurements of BNP to evaluate the relationship with changes in ECG findings showed that, in men, there was a significant correlation between a 5-year increase in BNP and changes in ECG findings. There was a clear gender difference between men and women. 
It is obvious that the measurement of BNP or long-term changes in BNP is not a substitute for ECG evaluation. Our findings suggest that men showing a 5-year increase in BNP of $10 \mathrm{pg} / \mathrm{ml}$ or more should be given subsequent detailed evaluations, including a ECG and echocardiography, with the aim of assessing the deterioration in cardiac function. No correlation between BNP and changes in ECG findings were observed in the women of our study; however, if the sample size were increased or the analysis were stratified for the use of medications or menstrual status, a correlation between increased BNP and changes in ECG findings might be identified. Further study is therefore needed.

Acknowledgments We are grateful to the individuals who participated in the official health checks in Sashiki-cho (in particular, Mr. Gentoku Tsuha, the mayor of Sashiki-cho at that time, now a part of Nanjo-shi), Okinawa for their cooperation with our analysis.

\section{References}

1. Heidenreich PA, Gubens MA, Fonarow GC, Konstam MA, Stevenson LW, Shekelle PG. Cost-effectiveness of screening with B-type natriuretic peptide to identify patients with reduced left ventricular ejection fraction. $\mathrm{J}$ Am Coll Cardiol. 2004;43(6):1019-26.

2. Morimoto T, Hayashino Y, Shimbo T, Izumi T, Fukui T. Is Btype natriuretic peptide-guided heart failure management costeffective? Int J Cardiol. 2004;96(2):177-81.

3. Sudoh T, Minamino N, Kangawa K, Matsuo H. Brain natriuretic peptide-32: N-terminal six amino acid extended form of brain natriuretic peptide identified in porcine brain. Biochem Biophys Res Commun. 1988;155(2):726-32.

4. Iwanaga Y, Nishi I, Furuichi S, Noguchi T, Sase K, Kihara Y, et al. B-type natriuretic peptide strongly reflects diastolic wall stress in patients with chronic heart failure: comparison between systolic and diastolic heart failure $\mathrm{J}$ Am Coll Cardiol. 2006;47(4):742-8.

5. Lukowicz TV, Fischer M, Hense HW, Doring A, Strizke J, Riegger G, et al. BNP as a marker of diastolic dysfunction in the general population: importance of left ventricular hypertrophy. Eur J Heart Fail. 2005;7(4):525-31.

6. Vanderheyden M, Goethals M, Verstreken S, De Bruyne B, Muller K, Van Schuerbeeck E, et al. Wall stress modulates brain natriuretic peptide production in pressure overload cardiomyopathy. J Am Coll Cardiol. 2004;44(12):2349-54.

7. Cowie MR. BNP: soon to become a routine measure in the care of patients with heart failure? Heart 2000;83:617-8.

8. Redfield MM, Rodeheffer RJ, Jacobsen SJ, Mahoney DW, Bailey KR, Burnett JC Jr. Plasma brain natriuretic peptide concentration: impact of age and gender. J Am Coll Cardiol. 2002;40(5):976-82.

9. Matsuda M. Significance of serum BNP measurement in annual health examination (in Japanese). J Kyorin Med Soc. 1999;30:73-8.

10. Richards AM, Nicholls MG, Espiner EA, Lainchbury JG, Troughton RW, Elliott J, et al. B-type natriuretic peptides and ejection fraction for prognosis after myocardial infarction. Circulation. 2003;107:2786-92.

11. Hirayama A, Kusuoka H, Yamamoto H, Sakata Y, Asakura M, Higuchi Y, et al. Usefulness of plasma brain natriuretic peptide concentration for predicting subsequent left ventricular remodeling after coronary angioplasty in patients with acute myocardial infarction. Am J Cardiol. 2006;98(4):453-7.

12. Sutovsky I, Katoh T, Takayama H, Seino Y, Takano T, Hayakawa $H$. Brain natriuretic peptide reflects the severity of ventricular arrhythmia. J Arrhythmia. 2001;17:479-82.

13. Alehagen U, Lindstedt G, Leviin LA, Dahlstrom U. Risk of cardiovascular death in elderly patients with possible heart failure. B-type natriuretic peptide (BNP) and the aminoterminal fragment of ProBNP (N-terminal proBNP) as prognostic indicators in a 6-year follow -up of a primary care population. Int J Cardiol. 2005;100(1):125-33.

14. Ogawa K, Oida A, Sugimura H, Kaneko N, Nogi N, Hasumi M, et al. Clinical significance of blood brain natriuretic peptide level measurement in the detection of heart disease in untreated outpatients comparison of electrocardiography, chest radiography and echocardiography. Circ J. 2002;66(2):122-6.

15. Redfield MM, Rodeheffer RJ, Jacobsen SJ, Mahoney DW, Bailey $\mathrm{KR}$, Burnett Jr JC. Plasma brain natriuretic peptide to detect preclinical ventricular systolic or diastolic dysfunction: a community-based study. Circulation. 2004;109(25):3176-81.

16. McKie PM, Rodeheffer RJ, Catalotti A, Martin FL, Urban LH, Mahoney DW, et al. Amino-terminal pro-B-type natriuretic peptide and B-type natriuretic peptide: biomarkers for mortality in a large community-based cohort free of heart failure. Hypertension. 2006;47(5):874-80.

17. Nakamura M, Sakai T, Osawa M, Onoda T, Yonezawa S, Okayama A, et al. Comparison of positive cases for B-type natriuretic peptide and ECG testing for identification of precursor forms of heart failure in an elderly population. Int Heart J. 2005;46:47787.

18. Olav WN, Jørgen H, Jørgen FH. Strong prognostic value of combing N-terminal atrial peptide and ECG to predict death in heart patients from general practice. Heart. 2001;86:218-9.

19. Wang TJ, Larson MG, Levy D, Benjamin EJ, Leip EP, Omland T, et al. Plasma natriuretic peptide levels and the risk of cardiovascular events and death. N Engl J Med. 2004;350:655-63.

20. Kawabe H, Wainai Y, Saito I. Relationship between annual changes in concentration of plasma $\mathrm{B}$ type natriuretic peptide and metabolic syndrome-related factors-evaluation in an annual check-up for lifestyle related disease (in Japanese). Ningendock. 2005;20(4):655-61.

21. Chang AY, Abdullah SM, Jain T, Stanek HG, Das SR, McGuire DK, et al. Associations among androgens, estrogens, and natriuretic peptides in young women observations from the Dallas Heart Study. J Am Coll Cardiol. 2007;49(1):109-16.

22. Hedberg P, Lönnberg I, Jonason T, Nilsson G, Pehrsson K, Ringqvist $\mathrm{I}$, et al. Electrocardiogram and B-type natriuretic peptide as screening tools for left ventricular systolic dysfunction in a population-based sample of 75-year-old men and women. Am Heart J. 2004;148:524-9.

23. Kawakubo K. The echocardiogram result classification. Japan Heart Association Circulatory Disorders Database (in Japanese). 2005; 12. Available at: http://www.jhf.or.jp. Accessed 8 January 2006. 